\title{
Relação entre funcionalidade e letramento funcional em saúde em pessoas idosas
}

\author{
Association between functional capacity and functional health literacy among older adults \\ Relación entre funcionalidad y alfabetización funcional en salud en personas mayores
}

Recebido: 08/02/2021 | Revisado: 12/02/2021 | Aceito: 17/02/2021 | Publicado: 24/02/2021

Eliel de Oliveira Bandeira

ORCID: https://orcid.org/0000-0003-1038-1612 Universidade Federal do Rio Grande, Brasil

E-mail: bandeira.eliel@hotmail.com

Daiane Porto Gautério Abreu

ORCID: https://orcid.org/0000-0002-1125-4693 Universidade Federal do Rio Grande, Brasil E-mail: daianeporto@furg.br

Juliana Piveta de Lima

ORCID: https://orcid.org/0000-0002-2703-9189 Universidade Federal do Rio Grande, Brasil E-mail: julianapivettal@hotmail.com

Nídia Farias Fernandes Martins

ORCID: https://orcid.org/0000-0001-5652-1110

Universidade Federal do Rio Grande, Brasil

E-mail: nidinhahfernandes@ hotmail.com

Aline Neutzling Brum

ORCID: https://orcid.org/0000-0002-9686-9602

Universidade federal do Rio Grande, Brasil

E-mail: neutzling@ furg.br

\begin{abstract}
Resumo
Objetivos: Verificar se há relação entre funcionalidade e letramento funcional em saúde em pessoas idosas assistidas pela Estratégia da Saúde da Família. Método: Estudo exploratório-descritivo, de abordagem quantitativa do tipo transversal, realizado em dez unidades de cobertura da Estratégia da Saúde da Família, totalizando 350 pessoas idosas. Para a coleta de dados utilizaram-se um questionário sociodemográfico e a aplicação dos instrumentos validados para mensurar a funcionalidade e o letramento funcional em saúde. A coleta de dados ocorreu no período de julho a dezembro de 2017 e foram realizados a análise estatística descritiva e os testes qui-quadrado, ANOVA e de correlação de Spearman. Resultados: Não houve relação entre atividades básicas de vida diária e letramento funcional em saúde. Houve uma correlação positiva entre as pontuações nas escalas de atividades instrumentais de vida diária e letramento funcional em saúde. Conclusão: Há relação entre funcionalidade e letramento funcional em saúde no que diz respeito às atividades instrumentais de vida diária.
\end{abstract}

Palavras-chave: Idoso; Atividades cotidianas; Alfabetização em saúde; Estratégia de saúde da família.

\begin{abstract}
Objective: To verify whether there is an association between functional capacity and functional health literacy among older adults assisted by the Family Health Strategy. Method: Exploratory descriptive study with a cross-sectional quantitative approach, carried out in the coverage area of ten Family Health Strategy teams, with a total of 350 older adults. Data was collected through a socio-demographic questionnaire and the application of validated instruments to measure functional capacity and functional health literacy. Data was collected from July to December 2017. Descriptive statistical analysis and chi-square, ANOVA and Spearman correlation tests were performed. Results: There was no relationship between basic activities of daily living and functional health literacy. There was a positive correlation between the scores on the scales of instrumental activities of daily living and functional health literacy. Conclusion: There is an association between functional capacity and functional health literacy regarding instrumental activities of daily living.
\end{abstract}

Keywords: Aged; Activities of daily living; Health literacy; Family health strategy.

\section{Resumen}

Objetivos: Verificar si existe una relación entre la funcionalidad y la alfabetización funcional en salud en las personas de edad asistidas por la Estrategia de Salud Familiar. Método: estudio exploratorio descriptivo, con un enfoque transversal cuantitativo, realizado en diez unidades de cobertura de la Estrategia de Salud Familiar, con un total de 350 personas mayores. Para la recolección de datos, se utilizó un cuestionario sociodemográfico y la aplicación de instrumentos validados para medir la funcionalidad y la alfabetización funcional en salud. La recolección de datos se realizó de julio a diciembre de 2017 y se realizaron análisis estadísticos descriptivos y pruebas de correlación de chicuadrado, ANOVA y Spearman. Resultados: No hubo relación entre las actividades básicas de la vida diaria y la 
alfabetización funcional en salud. Hubo una correlación positiva entre los puntajes en las escalas de actividades instrumentales de la vida diaria y la alfabetización funcional en salud. Conclusión: Existe una relación entre la funcionalidad y la alfabetización funcional en salud con respecto a las actividades instrumentales de la vida diaria.

Palabras clave: Ancianos; Actividades diarias; Alfabetización en salud; Estrategia de salud familiar.

\section{Introdução}

A capacidade funcional conceitua-se como a competência de manter as habilidades físicas e mentais para uma vida independente e autônoma (Pereira et al, 2017). O desempenho das atividades de vida diária (AVDs) é utilizado como parâmetro para determinar o estado funcional do indivíduo. Didaticamente essas atividades são divididas em Atividades Básicas de Vida Diária (ABVDs); Atividades Instrumentais de Vida Diária (AIVDs) e Atividades Avançadas de Vida Diária (AAVDs) (Dias et al, 2014).

As ABVDs constituem um marcador de maiores níveis de comprometimento funcional, e um declínio na execução dessas atividades é precedido por alterações em outras tarefas que envolvem interações entre os indivíduos e destes com os recursos do ambiente. As AIVDs são tarefas mais complicadas do que as ABVDs, no que tange às exigências neuropsicológicas e à influência de fatores sociais, motivacionais e contextuais para manutenção da vida independente. As AAVDs são relacionadas à melhor avaliação cognitiva e manutenção de excelente qualidade de vida e um leve declínio no desempenho destas atividades poderia ser um marcador de declínio funcional futuro (Dias et al, 2014).

Um estudo que buscou quantificar a prevalência de limitações funcionais na população de baixa e média renda entrevistou pessoas idosas em 43 países e detectou que 33,3\% dos entrevistados tinham algum tipo de incapacidade. Os países de Comores e Bangladesh obtiveram os piores resultados, chegando acima de 50\% de incapacidade (Hosseinpoor et al, 2016). Na população idosa brasileira, a prevalência da incapacidade para realizar as AVDs, segundo metanálise de estudos realizados em todas as regiões do país, variou de 12,3 a 94,1\% para os homens e de 14,9 a 84,6\% em mulheres (Campo et al, 2016).

Diversos fatores podem influenciar a funcionalidade das pessoas idosas, um desses fatores é o Letramento Funcional em Saúde (LFS) (McDougall, Mackert, Becker, 2018). O LFS corresponde ao conhecimento e às competências que as pessoas têm para acessar, compreender e aplicar as informações em saúde para tomada de decisões na vida cotidiana em matéria de cuidados de saúde (Sorensen et al, 2012).

O LFS é um determinante social da saúde e deve ser reconhecido como um indicador no monitoramento do estado de saúde da população. Nesta era de novos conhecimentos científicos, mudanças na prestação de cuidados, aumento do consumismo e avanços tecnológicos contínuos, os pacientes precisam de um alto nível de LFS para e orientar e fazer escolhas informadas. Portanto, o LFS tornou-se uma questão política e um desafio de saúde pública que não deve ser subestimado (Bonacorsi et al, 2017).

Nos Estados Unidos, mais de um terço dos adultos, cerca de 80 milhões de pessoas, têm conhecimento limitado em saúde, tornando mais difícil ler, entender e aplicar informações de saúde (Hersh, Salzman, Snyderman, 2015). No Brasil, um estudo realizado no Norte do país, com 114 pessoas idosas, verificou que 73,7\% dos entrevistados tinham LFS inadequado, 10,5\%, LFS marginal e apenas 15,8\%, LFS adequado. Entre os condicionantes para estes números estavam o baixo nível de escolaridade e a presença de doenças crônicas como hipertensão e diabetes mellitus (Santos, Portella, 2016).

A compreensão das pessoas idosas sobre sua própria saúde pode melhorar a adesão aos tratamentos, a realização de comportamentos benéficos à saúde, promover a independência e a autonomia e, consequentemente, melhorar a sua funcionalidade. Desse modo, é relevante conhecer a relação da funcionalidade com o LFS para que também os profissionais de saúde possam estabelecer intervenções para melhorar o LFS e a funcionalidade das pessoas idosas, já que no Brasil há uma lacuna no conhecimento, visto que não existem estudos que explorem essa relação e os estudos estrangeiros abordam a relação, porém, não de forma direta. Diante disso, tem-se como questão de pesquisa: Existe relação entre funcionalidade e letramento 
funcional em saúde em pessoas idosas assistidas pela Estratégia de Saúde da Família? Tem-se por objetivo: Verificar se há relação entre funcionalidade e letramento funcional em saúde em pessoas idosas assistidas pela Estratégia de Saúde da Família.

\section{Metodologia}

Estudo exploratório-descritivo, de abordagem quantitativa do tipo transversal, de acordo com a caracterização de Pereira et al. (2018), realizado em área de cobertura de dez unidades de saúde da Estratégia de Saúde da Família, totalizando 17 equipes que formam a zona oeste de um município do Sul do Brasil.

A população do estudo se constituiu de pessoas idosas (60 anos ou mais, conforme o Estatuto do Idoso) (Brasil, 2003) adscritas às equipes.

Os critérios de inclusão dos participantes foram: possuir pelo menos 1 ano de escolaridade autorreferida; conseguir ler o Cartão de Jaeger em nível 20/40, considerado normal para uma visão periférica com ou sem lentes corretoras ou óculos; ouvir o sussurro dos lados direito e esquerdo do canal auricular pelo teste do sussurro (Teste de Whisper); e conseguir pontuação adequada no Miniexame do Estado Mental (MEEM). Esses critérios são requisitos para a aplicação do instrumento de Letramento Funcional em Saúde.

Os critérios de exclusão foram: estar em tratamento com quimioterápicos ou com radioterapia ou ter sido submetido a procedimento cirúrgico nos últimos 15 dias anteriores à coleta de dados, pois essas condições podem interferir na funcionalidade.

Para definição da amostra, primeiramente calculou-se a população esperada para a área de abrangência do estudo. Assim, foi utilizado como referência o número máximo de pessoas a serem assistidas por equipe, que é de 4.000 pessoas (Brasil, 2012). Obteve-se um total de 68.000 pessoas (17 equipes $\mathrm{x} 4.000$ pessoas $=68.000)$.

O cálculo da população das pessoas idosas estimadas para a área de abrangência do estudo foi realizado tendo por base o percentual de idosos do município, que de acordo com o último censo demográfico realizado era de 13,89\% (Brasil, 2010). A partir da população das 17 equipes incluídas na pesquisa (68.000), foi calculada a porcentagem de pessoas idosas (13,89\%), obtendo-se como população do estudo aproximadamente 9.445 pessoas idosas.

Utilizou-se a fórmula de cálculo de amostras para população finita (Miot, 2011). Onde: Z - nível de significância do estudo $=95 \%(1,96) ; \mathrm{P}-$ prevalência do evento $=50 \%$ (utilizou-se 50\%, que maximiza o tamanho da amostra quando não se sabe a prevalência); $\mathrm{Q}$ - complementar da prevalência (1-P); N - população total = 9445 pessoas idosas; $\mathrm{E}$ - erro amostral de $5 \%$. Aplicando-se a fórmula, obteve-se $n=370$. A amostra foi por conveniência, e a seleção dos participantes foi feita por equipe: dividindo o n pelas 17 equipes participantes $(370 / 17=21,76)$, obtendo-se 22 pessoas idosas por equipe. Esse número foi ainda dividido pelo número de microáreas de cada equipe.

Realizou-se visita domiciliar para a coleta de dados às 376 pessoas idosas. Destas, 26 não foram incluídas no estudo, 18 por não atingirem a pontuação adequada no MEEM, seis por reprovação no teste de visão (Cartão de Jaeger) e duas por reprovação no teste do sussurro, utilizados como critérios de inclusão, obtendo-se como amostra final 350 pessoas idosas com uma perda amostral de 5,4\%. Os achados foram repassados às unidades de saúde por intermédio dos Agentes Comunitários de Saúde (ACS) para futuros encaminhamentos e cuidados de saúde com essas 26 pessoas idosas.

A coleta de dados foi realizada por três estudantes do curso de graduação em Enfermagem e três mestrandos do curso de mestrado em Enfermagem que receberam treinamento prévio no mês de junho de 2017.

Aplicou-se um questionário de caracterização sociodemográfica e de saúde elaborado para esta pesquisa. Para avaliar o LFS utilizou-se a versão breve do TOFHLA, por ser um instrumento completo e validado (Carthery-Goulart, 2009) e que verifica não apenas a compreensão de leitura, mas também o numeramento. A pontuação total do teste é de 100 pontos. Indivíduos pontuando entre zero e 53 pontos são considerados na faixa inadequada; entre 54 e 66 pontos, na faixa marginal; e entre 67 e 100 , na faixa adequada. 
Para mensurar a funcionalidade utilizou-se o Índice de Katz para as ABVDs por ser um instrumento validado no Brasil, de uso rápido, de manejo simples e fácil uso (Duarte, Andrade, Lebrão, 2007). Além de apresentar as atividades de maneira hierárquica pela sua complexidade. O instrumento possui seis questões, a pontuação total do instrumento é de 6 pontos, considerando a pessoa idosa como independente. De 4 a 6 pontos é considerada uma dependência moderada e abaixo de 2 pontos, dependência total (Duarte, Andrade, Lebrão, 2007).

Empregou-se a escala de Lawton e Brody para as AIVDs, por ser um instrumento de rápida utilização e fácil aplicabilidade, além de englobar atividades referentes à assistência, à qualidade da execução e à iniciativa. Esta escala contém nove itens e apresenta pontuação que varia entre 9 e 27 pontos. Pontuação menor ou igual a 5 pontos indica dependência total, pontuação maior que 5 e menor que 21 pontos, dependência parcial, e igual ou superior a 21 pontos representa independência para AIVDs (Brasil, 2007).

Foram realizados testes piloto com cinco pessoas idosas fora da área de cobertura das unidades selecionadas, aplicandose todos os instrumentos incluídos nesta pesquisa. O tempo médio de duração da aplicação dos instrumentos foi de 20 minutos, e não houve necessidade de alterações nos instrumentos. Os dados obtidos no teste piloto não integraram a amostra desta pesquisa.

A coleta de dados ocorreu no período de julho a dezembro de 2017. As pessoas idosas foram identificadas nas equipes a partir dos cadastros dos ACS, que indicaram os participantes de cada microárea que eram alfabetizados e conduziram os coletadores até as residências dos mesmos. Seguiu-se a lista dos ACS, se a pessoa idosa não estava na residência, ou não tinha interesse em participar da pesquisa, realizava-se a visita ao próximo constante na lista. As listas eram organizadas por endereços.

Para a organização dos dados foi elaborada uma planilha no programa Microsoft @ Excel, contendo um dicionário (codebook) e uma planilha que foi utilizada para digitação. Essa planilha foi verificada quanto à inconsistência dos dados. Logo após, foi transferida para o software Statistical Package for the Social Sciences (SPSS) versão 20.0.

Para análise quantitativa elaborou-se um banco de dados por meio da digitalização dos resultados no software SPSS versão 20.0. Foram realizadas: uma análise estatística descritiva, com descrição da frequência absoluta e frequência relativa para as variáveis categóricas e com uso das medidas de tendência central (média) e medidas de dispersão (desvio padrão) para variáveis numéricas; uma análise estatística inferencial, através de testes estatísticos para verificar a associação entre as variáveis.

O teste de Kolmogorov-Smirnov foi aplicado para verificação da normalidade dos dados numéricos e os testes estatísticos para comparação entre a variáveis foram definidos a partir da normalidade ou não dos dados.

Para verificar a associação entre a funcionalidade para ABVDs (categorizada em dependência total, moderada ou independência) e AIVDs (categorizada em dependência total, moderada ou independência) e o LFS (categorizada adequado, marginal ou inadequado) foi utilizado o teste de Qui-Quadrado. Para verificar a existência de diferença de média no grau de LFS entre os diferentes níveis de dependência para ABVDs (dependência total, moderada e independência) e AIVDs (dependência total, moderada e independência) foi utilizado o teste ANOVA. A correlação entre a pontuação nas escalas (Katz, Lawton e Brody e LFS) foi analisada por meio de Coeficiente de Correlação de Pearson. Em todos os testes foi considerado como estatisticamente significante o valor de $\mathrm{p}<0,05$.

O estudo obteve parecer favorável do Comitê de Ética em Pesquisa da Universidade Federal do Rio Grande, sob o número de 93/2017 e do Núcleo Municipal de Educação e Saúde Coletiva do município no qual foi realizado sob o número 013/2017, por se tratar de uma pesquisa em área de cobertura da Atenção Primária em Saúde. E seguiram-se as orientações da Resolução 466/2012, do Conselho Nacional de Saúde (Brasil, 2003), que diz respeito à pesquisa com seres humanos. Foi fornecido o Termo de Consentimento Livre e Esclarecido aos participantes. 


\section{Resultados}

Participaram do estudo 350 pessoas idosas. Dessas, a maioria tinha entre 60 e 69 anos 224 (64,0\%), eram do sexo feminino $236(67,4 \%)$, com companheiro $192(54,9 \%)$, com mais de quatro anos de estudo $182(52,0 \%)$, brancas 231 (66,0\%) e possuíam uma renda familiar acima de um salário mínimo 219 (62,8\%), conforme a Tabela 1.

Tabela 1: Distribuição das pessoas idosas de acordo com as variáveis sociodemográficas, Brasil, 2017.

\begin{tabular}{c|c}
\hline \multicolumn{1}{c|}{ Variáveis } & \multicolumn{1}{c}{$\mathbf{n}(\mathbf{\%})$} \\
sociodemográficas & $\mathbf{3 5 0}(\mathbf{1 0 0})$ \\
\hline Faixa etária & $224(64,0)$ \\
$60-69$ & $106(30,3)$ \\
$70-79$ & $20(5,7)$ \\
$\geq 80$ & $236(67,4)$ \\
Sexo & $114(32,6)$ \\
Feminino & $158(45,1)$ \\
Masculino & $192(54,9)$ \\
Estado marital & \\
Sem companheiro & $168(48)$ \\
Com companheiro & $182(52)$ \\
Escolaridade & \\
Até 4 anos & $231(66)$ \\
$>4$ anos & $119(34)$ \\
Cor & \\
Branco & $130(37,2)$ \\
Não branco & $219(62,8)$ \\
Renda* & Fonte: Autores $(2017)$. \\
$\leq 1$ salário &
\end{tabular}

Entre as pessoas idosas entrevistadas, $295(84,3 \%)$ eram independentes, 48 (13,7\%) tinham dependência moderada e 7 (2\%) tinham dependência total para as ABVDs. Em relação às AIVDs, 329 (94\%) eram independentes, enquanto que 21 (6\%) tinham dependência moderada. Não houve participantes com dependência total para as AIVDs. Em relação ao LFS, 86 (24,5\%) tinham letramento adequado, 58 (16,6\%) tinham letramento marginal e $206(58,9)$ tinham letramento inadequado.

A Tabela 2 apresenta a associação entre os níveis de dependência das atividades de vida diária e os níveis de LFS. Para os diferentes níveis de ABVDs, a maioria tinha o LFS inadequado. Contudo, o percentual de LFS inadequado foi maior para aqueles com dependência total [,] seguidos daqueles com dependência moderada e, depois, os independentes. Não houve relação significativa estatisticamente entre os níveis de ABVDs e os níveis de LFS.

Tabela 2: Associação entre Atividades de Vida Diária e LFS em pessoas idosas, Brasil, 2017.

\begin{tabular}{|c|c|c|c|c|c|c|}
\hline Atividades de Vida Diária & $\begin{array}{c}\text { LFS } \\
\text { adequado n } \\
(\%)\end{array}$ & $\begin{array}{l}\text { LFS } \\
\text { marginal n } \\
(\%)\end{array}$ & $\begin{array}{l}\text { LFS } \\
\text { inadequado } \mathrm{n} \\
(\%)\end{array}$ & $\begin{array}{l}\text { Total } \\
\text { n }(\%)\end{array}$ & $\mathbf{X}^{2}$ & $\begin{array}{l}\text { Valor } \\
\text { p }\end{array}$ \\
\hline \multicolumn{7}{|l|}{ ABVD } \\
\hline Independente & $76(25,7)$ & $49(16,6)$ & $170(57,7)$ & $295(100)$ & 5,570 & 0,234 \\
\hline Dependência moderada & $10(20,8)$ & $09(18,8)$ & $29(60,4)$ & $48(100)$ & & \\
\hline Dependência total & 0 & 0 & $07(100)$ & $7(100)$ & & \\
\hline \multicolumn{7}{|l|}{ AIVD } \\
\hline Independente & $85(25,8)$ & $53(16,1)$ & $191(58,1)$ & $329(100)$ & 4,874 & 0,087 \\
\hline Dependência parcial & $01(4,8)$ & $05(23,8)$ & $15(71,4)$ & $21(100)$ & & \\
\hline
\end{tabular}

Fonte: Autores (2017). 
Para os que eram independentes e tinham dependência parcial paras as AIVDs, a maioria tinha o LFS inadequado. Não houve relação significativa estatisticamente entre os níveis de AIVDs e os níveis de LFS, e aqueles que tinham dependência parcial apresentaram percentual maior de LFS inadequado do que os independentes.

A Tabela 3 apresenta uma análise por ANOVA das médias de LFS entre os níveis de dependência para as ABVDs e as AIVDs. Nas ABVDs, tanto os independentes, quanto os com dependência moderada obtiveram a mesma média no LFS (52,2), essa média foi bem superior em comparação aos que tinham dependência total $(35,0)$. Contudo, não houve diferença significativa estatisticamente entre as médias para ABVDs $(\mathrm{p}=0,125)$.

Em relação às AIVDs, os que eram independentes tinham uma média de LFS 52,5 maior do que aqueles com dependência parcial (42,5). Houve diferença significativa estatisticamente entre as médias ( $\mathrm{p}=0,040)$. As médias, tanto para ABVDs como para AIVDs, correspondem a um LFS inadequado.

Tabela 3: Médias do LFS de acordo com os níveis de ABVDs e AIVDs para as pessoas idosas, Brasil, 2017.

\begin{tabular}{lll|l|l|l}
\hline Variáveis & $\mathbf{n}(\%)$ & Média LFS & DP & F & P \\
\hline ABVDs & & & & 2,092 & 0,125 \\
Independência & $295(84,3)$ & 52,2 & 22,7 & & \\
Dependência moderada & $48(13,7)$ & 52,2 & 19,4 & & \\
Dependência total & $07(2,0)$ & 35,0 & 14,0 & & \\
AIVDs & & & & 4,262 & 0,040 \\
Independência & $329(94)$ & 52,5 & 22,3 & & \\
Dependência parcial & $21(6,0)$ & 42,2 & 17,5 & & \\
\hline
\end{tabular}

Fonte: Autores (2017).

Verificou-se que, conforme aumentava a pontuação na escala de LFS, também aumentava a pontuação na escala de AIVDs, sendo essa correlação significativa (rho- 0,152 p=0,004). Não houve correlação significativa estatisticamente para a relação do LFS com as ABVDs (rho=0,31 p=0,564), embora também tenha ocorrido uma correlação positiva entre as escalas. Houve uma correlação positiva entre a escala que mede ABVDs e a que mede AIVDs, o aumento em uma influenciando o aumento na outra $(\mathrm{rho}=0302$ e $\mathrm{p}=<0,001)$.

\section{Discussão}

O presente estudo revelou que as pessoas idosas assistidas pela Estratégia de Saúde da Família eram predominantemente do sexo feminino, com idade inferior a 80 anos, com companheiro; estes dados assemelham-se com estudo realizado em áreas de cobertura da Estratégia de Saúde da Família na Região Nordeste do Brasil (Pinto et al, 2016). A renda assemelha-se a outro estudo realizado com 388 pessoas idosas atendidas em Unidades de Saúde da Estratégia de Saúde da Família, porém, nas variáveis escolaridade e cor, houve divergência, em que apontou que a maioria nunca estudou ou estudou até quatro anos, e eram da cor parda (Pereira et al, 2017).

Nas ABVDs, a prevalência de independência funcional nas pessoas idosas foi de 84,3\% assemelhando-se a estudos realizados na Região Nordeste, na Região Sudeste e na Região Sul do Brasil (Pinto et al, 2016, Nunes et al, 2017), os quais encontraram que mais da metade das pessoas idosas tinha sua funcionalidade mantida. Entretanto, um estudo realizado nos Estados Unidos, mostrou que, de 1982 a 2004, houve um declínio na porcentagem de pessoas idosas com alguma limitação funcional, porém, de 2004 a 2011, esta porcentagem tornou a aumentar. Outro resultado dessa pesquisa mostrou que, no período 
de 2004 a 2011, houve aumento de mulheres idosas incapazes de comprar, administrar seu dinheiro ou lavar roupas sem ajuda (Freedman, Wolf, Spillman, 2016).

A prevalência de dependência nas AIVDs foi 21 (6\%), resultado diferente aos outros estudos no Brasil, em que a prevalência variou entre 34,2\% de dependentes na cidade de Bagé, no Estado do Rio Grande do Sul (Nunes et al, 2017), e 46,3\% de dependentes na cidade de Uberaba, no Estado de Minas Gerais (Virtuoso et al, 2015). Em relação ao LFS, 58,8\% das pessoas idosas tinham o LFS inadequado; esse resultado é semelhante ao de um estudo realizado na União Europeia, que constatou que cerca de 50\% da população não possuía LFS adequado (Sorensen et al, 2015).

Ao analisar a relação das AVDs com o LFS por níveis, constatou-se que não houve uma associação significativa estatisticamente, podendo estar relacionado ao fato de que, tanto nas pessoas idosas independentes, quanto nas que tinham alguma dependência, o nível de LFS foi inadequado. Isto reforça que a população idosa tem dificuldades em compreender e utilizar as informações em saúde, o que pode resultar em uma baixa capacidade para gerir a própria saúde e o processo de adoecimento, e também na baixa adesão às medidas de promoção da saúde e prevenção de doenças (Passamai et al, 2012).

A média do LFS das pessoas idosas do estudo correspondeu a um LFS inadequado, contudo, foi maior para os que eram independentes nas ABVDs do que para os que eram dependentes totais. Nas AIVDs também ocorreu diferença de média, tendo os independentes melhor média, mesmo com um LFS inadequado. A associação entre o LFS inadequado e a deficiência da capacidade funcional nessa faixa etária pode refletir uma história de acesso precário aos cuidados, que permeia a associação entre o letramento mais baixo e resultados de saúde mais pobres na população idosa (Brown et al, 2014).

Existem poucos estudos abordando a relação da funcionalidade com o LFS, porém, as estruturas teóricas e a pesquisa empírica fornecem suporte para uma relação hipotética entre o LFS e o declínio da função física (Smith et al, 2015). Em uma amostra de 2923 pessoas idosas inscritas no Managed Care da Medicare, os entrevistados com níveis mais baixos de LFS relacionavam-se com uma pior função física e saúde mental; uma probabilidade maior de reportarem dificuldades nas AVDs e as AIVDs; uma limitação maior na atividade devido a uma saúde física debilitada; menos realizações devido à frágil saúde física; e um maior número de relatos de dor que interfere nas AVDs (Wolf, Gazmararian, Baker, 2005).

Um caminho entre o LFS e os resultados de saúde pode se dar por meio de comportamentos de saúde como a prática de atividade física, consumo de frutas e vegetais, tabagismo, consumo de café da manhã, consumo de álcool e índice de massa corporal (IMC). As pessoas idosas com baixo LFS podem estar menos conscientes da importância de terem ou não certos comportamentos para manutenção ou melhoria do estado de saúde. Além disso, é necessário ter um nível adequado de letramento para tomar decisões apropriadas em relação aos comportamentos de saúde (Geboers et al, 2016).

O LFS leva a um melhor conhecimento dos riscos nos serviços de saúde e ao cumprimento das ações prescritas, capacidade melhorada para atuar de forma independente, motivação melhorada e mais autoconfiança, melhora a resiliência individual à adversidade social e econômica. Em termos de benefícios comunitários e sociais, o LFS aumenta a participação em programas de saúde da população, aumenta a capacidade de influenciar as normas sociais e interage com os grupos sociais, melhora o empoderamento da comunidade e aumenta a capacidade de atuar sobre os determinantes sociais e econômicos da saúde (Sorensen et al, 2012).

Neste estudo, somente as AIVDs tiveram associação com o LFS, porém, a predominância dos participantes foi de pessoas idosas na etapa inicial da velhice, sendo 64\% na faixa entre 60 e 69 anos, e as pessoas idosas nessa faixa etária têm melhores níveis de funcionalidade. Sabendo que as pessoas idosas tendem a perder primeiramente as AIVDs para depois perderem as ABVDs, e que houve relação entre o Índice de Katz, que mede as ABVDs, e o Lawton e Brody, que me as AIVDs, acredita-se que com o avançar da idade essas pessoas idosas irão se tornar mais dependentes para as ABVDs. Supõe-se, então, que o LFS exerce influência nas ABVDs de forma indireta. Contudo, são necessários mais estudos com populações de pessoas idosas em faixas etárias mais avançadas para comprovar ou descartar tais suposições. 


\section{Conclusão}

Não houve relação entre ABVDs e LFS. Houve relação entre as AIVDs e LFS, e as médias de LFS para quem apresentava dependência parcial foram menores do que as daqueles que eram independentes. Também houve uma correlação positiva entre as pontuações nas escalas de AIVDs e LFS.

Acredita-se que os resultados encontrados neste estudo contribuem para o avanço do conhecimento sobre LFS e funcionalidade em pessoas idosas, tendo em vista a escassez de estudos na área. Os resultados do estudo mostram a importância da avaliação da relação entre LFS e funcionalidade para planejamento da assistência integral à pessoa idosa no contexto da Estratégia de Saúde da Família.

Este estudo mostrou por meio de seus resultados, que embora a população idosa está mais independente, o letramento funcional em saúde pode influenciar a autonomia e a independência dessa população. Por isso a importância de uma adequada educação em saúde para a promoção de saúde e prevenção de doenças, e a relevância de mais estudos sobre essa relação, visto que essa temática ainda é incipiente no Brasil.

\section{Referências}

Bonacorsi, G., Grazzini, M., Pieri, L., Santomauro, F., Ciancio, M., \& Lorini, C. (2017). Assessment of Health Literacy and validation of single-item literacy screener (SILS) in a sample of Italian people. Ann Ist Super Sanità, 53(3), 205-212.

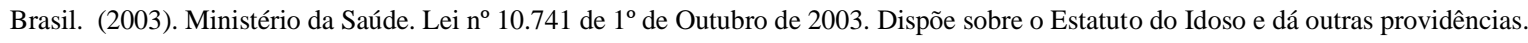

Brasil. (2007). Ministério da Saúde. Envelhecimento e saúde da pessoa idosa. Brasília.

Brasil. (2003). Ministério da Saúde. Resolução CNS 466 de 12 de dezembro de 2012. Diretrizes e normas. regulamentadoras de pesquisas envolvendo seres humanos. Conselho Nacional de Saúde.

Brasil. (2010). Instituto Brasileiro de Geografia e Estatística. Banco de Dados. Cidades@. Informações sobre os municípios Brasileiros. RS. Rio Grande. Censo demográfico 2010: resultados da amostra - características da população.

Brasil. (2012). Ministério da Saúde. Política Nacional de Atenção Básica. Brasília.

Brown, R. T., Pierluissi, E., Guzman, D., Kessell, E.R., Goldman, E., \& Sarkar, U., et al. (2014). Functional disability among late middle-aged and older adults admitted to a safety-net hospital. J Am Geriatr Soc, 62(11), 2056-63.

Campos, A. C. V., Almeida, M. H. M., Campos, G. V., \& Bogutchi, T. F. (2016). Prevalence of functional incapacity by gender in elderly people in Brazil: a systematic review with meta-analysis. Rev Bras Geriatr Gerontol, 19(3), 545-59.

Carthery-Goulart, M. T., Anghinah, R., Areza-Fegyveres, R., Bahia, V. S., \& Brucki, S. M. D., et al. (2009). Performance of a Brazilian population on the test of functional health literacy in adults. Rev Saúde Públ, 43(4), 631-38.

Dias, E. G., Duarte, Y. A. O., Almeida, M. H. M., \& Lebrão, M. L. (2014). The advanced activities of daily living at component of the functional assessment in elderly people. Rev Ter Ocup Univ., 25(3), 225-32.

Duarte, Y. A. O., Andrade, C. L., \& Lebrão, M. L. (2007). Katz Index on elderly functionality evaluation. Rev Esc Enferm USP, 41(2), 317-25.

Freedman, V. A., Wolf, D. A., \& Spillman, B. C. (2016). Disability-Free Life Expectancy Over 30 Years: A Growing Female Disadvantage in the US Population. Am J Public Health, 106(6), 1079-85.

Geboers, B., Reinjneveld, S. A., Jansen, C. J., \& de Inverno, A. F. (2016). Health Literacy Is Associated with Health Behaviors and Social Factors Among Older Adults: Results from the LifeLines Cohort Study. J Health Commun.,21(2), 45-53.

Hersh, L., Salzman, B., \& Snyderman, D. (2015). Health Literacy in Primary Care Practice. Am Fam Physician, 92(2), 118-124.

Hosseinpoor, A. R., Bergen, N., Kostanjsek, N., Kowal, P., Oficial, A., \& Chatterji, S. (2016). Socio-demographic patterns of disability among older adult populations of low-income and middle-income countries: results from World Health Survey. Int J Public Health, 61(3), 337-45

McDougal, G. J., Mackert, H., \& Becker, H. (2012). Memory Performance, Health Literacy, and Instrumental Activities of Daily Living of Community Residing Older Adult. Nurs Res, 61(1), 70- 75.

Miot, H. A. (2011). Sample size in clinical and experimental trials. J Vasc Bras, 10(4), 275-278.

Nunes, J. D., Saes, M. O., Nunes, B. P., Siqueira, F. C. V., Soares, D. C., \& Fassa, M E. G., et al. (2017). Functional disability indicators and associated factors in the elderly: a population-based study in Bagé, Rio Grande do Sul, Brazil. Epidemiol Serv Saúde, 26(2), 295-304.

Passamai, M. P. B., Sampaio, H. A. C., Dias, A. M. I., \& Cabral., L. A. (2012). Functional Health Literacy: Reflections and concepts on its impact on the interaction among users, professionals and the health system. Interface (Botucatu), 16(41), 301-314. 
Research, Society and Development, v. 10, n. 2, e48210212775, 2021

(CC BY 4.0) | ISSN 2525-3409 | DOI: http://dx.doi.org/10.33448/rsd-v10i2.12775

Pereira, L. C., Figueiredo, M. L. F., Belez,a C. M. F., Andrade, E. M. L. R., Silva, M. J., \& Pereira, A. F. M. (2017). Predictors for the functional incapacity of the elderly in primary health care. Rev Bras Enf., 70(1), 112-18.

Pereira, A. S. et al. (2018). Metodologia da pesquisa científica. UAB/NTE/UFSM. https://repositorio.ufsm.br/bitstream/handle/1/15824/ Lic_Computacao_Metodologia-Pesquisa-Cientifica.pdf?sequence=1

Pinto Junior, E. P., Silva, I. T., Vilela, A. B., Casotti, C. A., Pinto, F. J. M., \& Silva, M. G. C. (2016). Functional dependency and associated factor in elderly living with relatives. Cad Saúde Colet, 24(4), 404-12.

Santos, M. I. P. O., \& Portella, M. R. (2016). Conditions of functional health literacy of an elderly diabetics group. Rev Bras Enf, 69(1), 156-64.

Smith, S. G. et al. (2015). Low health literacy predicts decline in physical function among older adults: findings from the LitCog cohort study. J Epidemiol Community Health, (69), 474-80.

Sorensen, K. et al, (2012). Health literacy and public health: a systematic review and integration of definitions and models. BMC Public Health, 12(80), 1-13. https://doi.org/10.1186/1471-2458-12-80

Sorensen, K. et al. (2015). Health literacy in Europe: comparative results of the European health literacy survey (HLS-EU). Eur J Public Health, 25(6), 10531058 .

Virtuoso Junior, J. S., Martins, C. A., Roza, L. B., Paulo, T. R. S., Ribeiro, M. C. L., \& Tribess, S. (2015). Prevalence of disability and associated factors in the elderly. Texto Contexto, 24(2), 521-9.

Wolf, M. S., Gazmararian, J. A., Baker, D. W. (2005) Health literacy and functional health status among older adults. Arch Inter, 165(17), 1946-52. 